Ann. Biol. anim. Bioch. Biophys., I975, 15 (I), 9-18.

\title{
RÉACTION CORTICALE ET BLOCAGE DE LA POLYSPERMIE DANS L'CEUF DE LAPINE
}

\author{
J.-E. FLÉCHON, D. HUNEAU, Aline SOLARI et C. THIBAULT \\ Station centrale de Physiologie animale, \\ Centre national de Recherches zootechniques, I. N. R. A., \\ 78350 Jouy en Josas
}

\section{RÉSUMÉ}

Des observations en microscopie électronique et des analyses biométriques ont été effectuées sur les granules corticaux $(\mathrm{GC})$ de différentes catégories d'œufs de lapines.

Les résultats obtenus montrent que les GC demeurent présents dans le cortex des cufs ovulés pendant leur période de fécondabilité (8 à ıo heures après l'ovulation). Il en est de même dans les oufs activés artificiellement par un refroidissement de 24 heures à $10^{\circ} \mathrm{C}$ puis réchauffement; ces œufs restent fécondables.

Dans les oufs fécondés et les oufs activés puis fécondés, les GC ont pratiquement disparu. C'est la pénétration du spermatozoïde fécondant qui provoque l'émission des GC et en retour, cette réaction corticale inhibe la fusion avec l'œuf de spermatozoïdes surnuméraires. Le rôle des GC dans la fécondation semble être restreint à cette fonction.

Le nombre des GC a tendance à diminuer dans les ovocytes âgés, activés ou non, ce qui explique le risque accru de polyspermie en cas de fécondation tardive après l'ovulation.

\section{INTRODUCTION}

La prévention de la polyspermie chez les Mammifères s'effectue d'abord dans les voies génitales par une limitation du nombre de spermatozoïdes qui atteignent le lieu de fécondation (Thibaul,T, I972). Au niveau de l'œuf, deux systèmes contribuent à cette limitation : la zone pellucide et la membrane plasmique. Les granules corticaux (GC) jouent un rôle actif dans cette régulation (PIKo, I969).

L'argument le plus solide pour appuyer cette proposition est de mettre en évidence la possibilité de fécondation tant que les granules restent présents et l'impossibilité quand ils ont été émis. 
Nous nous sommes adressés à l'œuf de Lapine, lequel, après activation parthénogénétique, reste fécondable (CHAMEL, I962), et constitue un excellent modèle pour l'étude du problème posé. Tel est l'objet du présent mémoire.

\section{MATÉRIEL, ET MÉTHODES}

Des ovocytes fraîchement ovulés de lapines sont activés par un séjour de 24 heures à I $0^{\circ} \mathrm{C}$ dans du sérum homologue et une remise en culture à $37^{\circ} \mathrm{C}$ (CHALMEL, 1962 d'après $\mathrm{CHANG}_{\text {H }}$, I954).

Ces œufs sont partagés entre trois lots expérimentaux :

I. Culture seule in vitro à $37^{\circ} \mathrm{C}:$ " œufs activés in vitro ".

2. Transplantation par laparatomie dans un pavillon d'une femelle mise au mâle vasectomisé Io heures auparavant et dont les follicules mûrs sont cautérisés, puis récupération des œufs transplantés par perfusion des oviductes 4 à 6 heures après : cufs activés in vivo ».

3. Même traitement mais avec une femelle accouplée avec un mâle normal : " ceufs activés et fécondés in vivo ".

Sont également étudiés, des œufs vierges ou fécondés recueillis I à Io heures après l'ovulation. Tous les oufs subissent une double fixation (glutaraldéhyde puis tétroxyde d'osmium), une déshydratation dans du méthanol additionné d'acétate d'uranyle, puis une inclusion dans l'Epon. Les coupes sont contrastées par le citrate de plomb après traitement par l'acétate d'uranyle dans certains cas.

Les observations des coupes sont complétées par un comptage des CG sur des sections paraéquatoriales des œufs. Les oufs activés in vivo n'ont pas été utilisés pour l'analyse statistique.

A partir du nombre moyen de GC par unité de longueur, nous avons calculé pour chaque catégorie les moyennes, variances, coefficient de variation et les intervalles de confiance non paramétriques au seuil i p. roo. La comparaison, deux à deux, des différentes catégories a été effectuée avec le test $U$ de Mann-Whitney.

\section{Définition des catégories d'aufs}

Sont considérés comme activés tous les aufs conservés 24 h à $10^{\circ} \mathrm{C}$ et ramenés à $37^{\circ} \mathrm{C}$ in vitro ou in vivo. Ce traitement est efficace à $100 \mathrm{p}$. Ioo et provoque une segmentation avec présence le plus souvent de deux pronuclei, l'un formé par les chromosomes de l'ovule et l'autre par ceux du deuxième globule polaire non émis (CHALMEL, I962).

Sont classés activés puis fécondés, tous les œufs dans le cytoplasme desquels a été observée la pénétration d'un spermatozoïde (au moins 70 p. Ioo des œufs activés in vivo chez des femelles accouplées à un mâle normal, CHALmel, I962). Sont exclus les œufs où des spermatozoïdes n'ont pu être observés que dans la zone pellucide ou l'espace périovulaire.

\section{RÉSULTATS}

\section{Observations}

Les oufs activés in vitro ou in vivo possèdent des GC dont la distribution et l'aspect paraissent semblables à ceux d'œufs ovulés non fécondés (fig. I et 2). Les $\mathrm{GC}$ forment sous la membrane plasmique une assise monogranulaire discontinue, interrompue à l'endroit où le premier globule polaire s'est détaché.

Dans les œufs activés artificiellement, en particulier, les GC sont parfois distribués localement de manière irrégulière dans le cortex (comparer les fig. 3 et 4).

Les cufs activés puis fécondés in vivo présentent un cortex analogue à celui d'œufs fécondés, c'est-à-dire pratiquement dépourvu de GC (fig. 5 et 6). 
On observe différents aspects de la pénétration du spermatozoïde fécondant dans des oufs activés: dans certains cas, le noyau évolue normalement et commence à gonfler alors que dans d'autres cas où le spermatozoïde semble pourtant à un stade de pénétration avancé, aucune modification du noyau ne se produit.

\section{Analyse biométrique}

L'estimation des droites de régression entre le nombre de GC et la longueur de cortex (graph. I), la signification des coefficients de corrélation (tabl. I) et la comparaison du nombre moyen de GC par unité de longueur de cortex (graph. I) permettent de bien séparer les œufs fécondés des œufs non fécondés.

TABLEAU I

Représentation des tests de comparaison des différentes catégories d'aufs

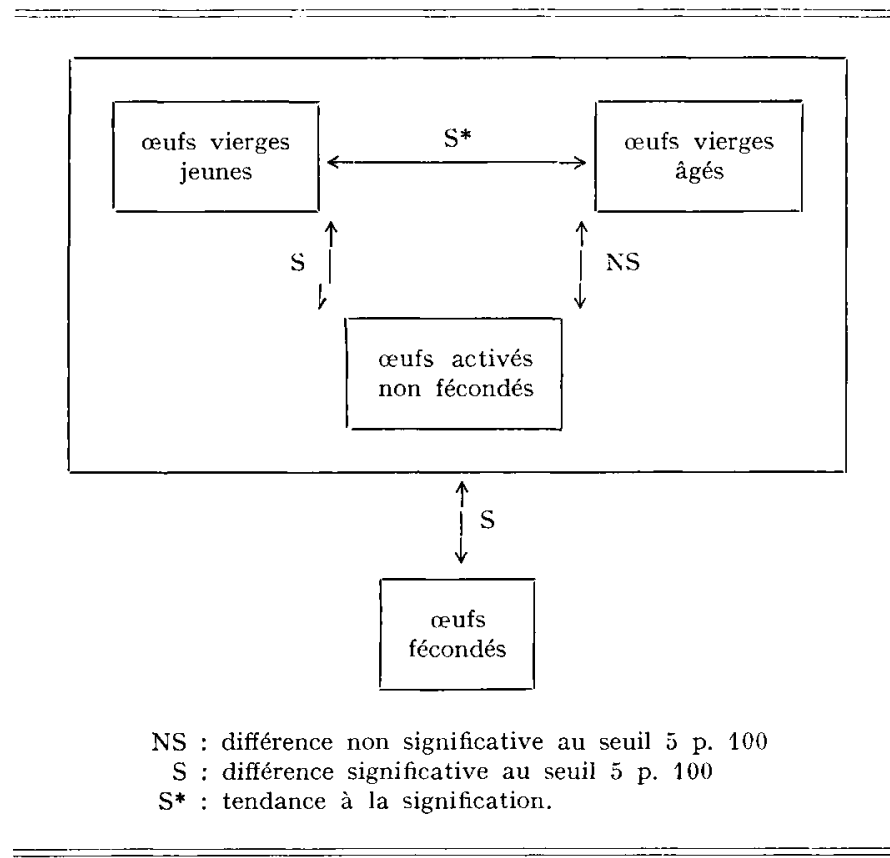

Pour les œufs non fécondés les corrélations diffèrent significativement de zéro : le nombre de GC serait proportionnel à la longueur de cortex observée. Cependant, nous ne pouvons tester la linéarité de la relation en raison du faible nombre de données, mais les valeurs élevées des coefficients constituent un argument en faveur de la valadité de cette hypothèse.

Les œufs activés non fécondés s'apparentent aux œufs vierges âgés ( $\geqslant$ I $8 \mathrm{~h}$ post-coitum) et montrent une différence significative avec les œufs vierges jeunes ( $\leqslant$ I4 h 30 post-coitum). La différence entre œufs vierges jeunes et âgés est à peine 
significative; elle serait très significative $(\mathrm{P}<\mathrm{I}$ p. Ioo) si le lot des vierges jeunes ne comportait pas un œuf (exceptionnel) présentant un faible nombre de GC par unité de longueur de cortex.

Les œufs activés présumés fécondés constituent une population hétérogène. Les œufs activés dont le cytoplasme est pénétré par un spermatozoïde (œufs activés puis fécondés), sont identiques aux œufs fécondés. Celui de ces œufs qui a conservé le plus de $\mathrm{GC}$ se trouve encore au stade initial de la fusion des gamètes.
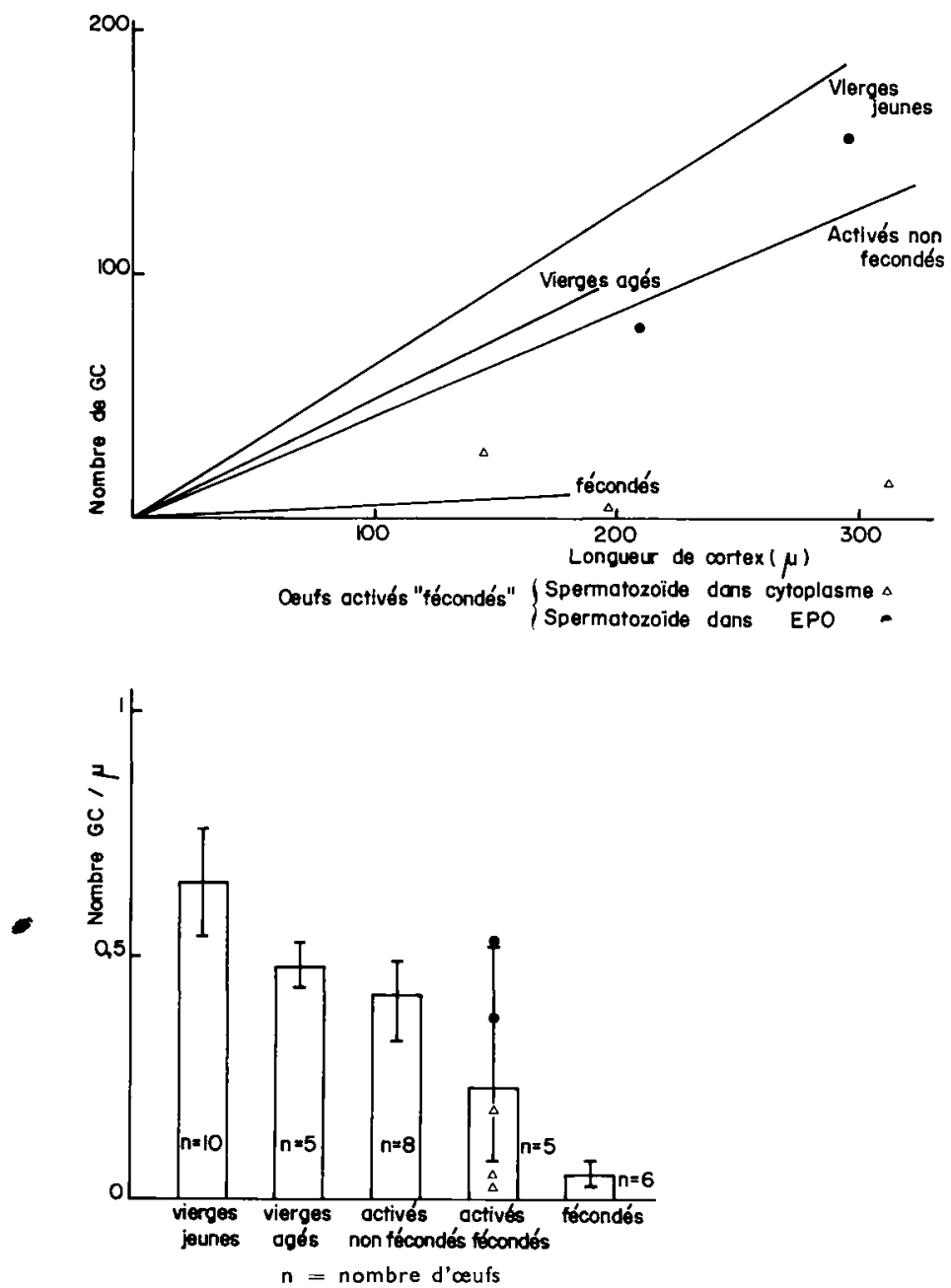

GRAPH. I. - Nombre de granules corticaux pour les différentes catégories d'œufs.

haut : droite de régression

bas : valeur par unité de longueur

Pour les œufs dans lesquels on n'a trouvé un spermatoïde que dans 1'espace périovulaire (EPO), le nombre de GC par unité de longueur de cortex s'apparente à celui des œufs activés non fécondés (graph. I). 


\section{DISCUSSION}

\section{('ritiques des méthodes biométriques utilisées}

L'œuf et les GC peuvent être assimilés respectivement à une grande sphère à la face interne de laquelle sont situées, en position tangentielle, de très nombreuses petites sphères égales. Dans ces conditions, une estimation relative de la densité des GC par unité de surface peut-être donnée par le nombre de GC rapporté au périmètre d'une section équatoriale.

Nous n'avons jamais observé de gradient de distribution des GC dans le cortex sur des coupes équatoriales d'œufs ; dans le cas contraire, le calcul du nombre moyen par unité de longueur ne serait pas correct. En fait, l'augmentation linéaire du nombre de $\mathrm{GC}$ en fonction de la longueur de cortex des œufs vierges est en faveur de leur répartition relativement homogène.

Une erreur absolue sur ce nombre est introduite par les variations éventuelles de l'épaisseur des coupes, qui est inférieure à celle des GC (de l'ordre respectivement de $0, \mathrm{I}$ et $0,25 \mu$ ). Cependant, l'épaisseur des coupes est semblable pour les différentes catégories d'œufs, pour lesquelles l'erreur éventuelle est ainsi identique et il n'y a done pas lieu d'en tenir compte.

\section{Devenir et rôle des granules corticaux}

Les nombres moyens de GC par unité de longueur confirment l'impression donnée par les observations, à savoir que les œufs vierges et activés d'une part et les œufs fécondés et activés ptiis fécondés d'autre part, constituent deux populations distinctes.

Les œufs ovulés non fécondés conservent des GC au moins pendant la période de fécondabilité (jusqu'à I 8-20 h post-coitum, âge maximum des œufs vierges utilisés ici) mais leur fréquence diminue dans le cortex. Chez la Ratte, si la décharge ovulante est retardée de 48 heures, le nombre de GC des ovocytes finalement ovulés, diminue de 50 p. Ioo (Peluso et Butcher, I974). A la fin de la période de fécondabilité, il est possible que les GC soient émis spontanément (Hamster : YaNAGimachi et Chang, I96I ; Souris : MERchan'T et Chang, I97I) ou bien qu'ils forment des agregats (LONGO, I974a) ou migrent vers le centre de l'œuf (Souris : SzöL_LösI, I97I). Leur absence du cortex pourrait expliquer des anomalies de fécondation tardive (Austin, I970).

Les œufs activés in vitro ou in vivo par refroidissement gardent aussi des GC jusqu'à $6 \mathrm{~h}$ après réchauffement (FLÉcHON, I970), de même que les œufs de Hamster activés naturellement ou artificiellement (AUSTIN, I956 Longo, I974 b). Mais, comme dans les œufs vierges âgés, le nombre de $\mathrm{GC}$ a tendance à diminuer. $\mathrm{Au}$ contraire, l'activation par choc électrique (TARkovski et al., 1970) provoquerait une émission de GC (Gwatkin et al., I973).

Dans les œufs fécondés, l'émission des GC est massive mais si elle n'est pas toujours totale, la perte de la plupart des GC par le zygote a été maintes fois confirmée (PIko, I969) et a été utilisée comme test de fécondation in vitro (FRASER et al., I972).

Il semble évident que les œufs activés puis fécondés, selon la définition donnée 
plus haut, c'est-à-dire dont le cytoplasme a bien été pénétré par un spermatozoïde, émettent leurs GC d'une manière analogue aux œufs fécondés. Un barrage contre la polyspermie se produit effectivement dans les œufs activés puis fécondés puisque 7 p. Ioo d'entre eux seulement sont polyspermiques, taux normal pour des cufs âgés (Chalmei, I 962). De même, chez le Rat, les œufs activés (émission du deuxième globule polaire) restent fécondables et la pénétration d'un spermatozoïde fécondant bloque normalement la polyspermie dans ces œufs (AUSTIN et BRADEN, r954).

\section{Mécanisme d'action du matériel cortical dans le blocage de la polyspermie}

Les granules corticaux de l'œuf de Lapine sont constitués de glycoprotéines ne présentant pas de groupes acides libres, en quantité décelable (FIÉcHON, I970). Cette composition ne se modifie pas dans les cufs non fécondés (vierges âgés ou activés).

Dans le cas de blocage essentiellement au niveau de la membrane plasmique (lapine), les glycoprotéines des GC peuvent réagir avec cette dernière et modifier la surface cellulaire (COOPER et BEDFORD, I97I).

Lorsque les spermatozoïdes surnuméraires sont arrêtés partiellement ou totalement dans la zone pellucide, la " réaction " de cette dernière serait due à l'action de la substance des GC sur la pellucide (AUSTIn et Braden, I956; Barros et YanAGIMACHI, I97I).

Chez la Lapine où se produit aussi un arrêt partiel des spermatozoïdes dans la zone pellucide, cette dernière est plus résistante à une digestion par la trypsine après fécondation et 1'hypothèse d'une libération d'inhibiteur de trypsine par les GC a été avancée (CoNRAD et al., I97I).

Chez la Souris où le nombre de spermatozoïdes surnuméraires dans l'espace périovulaire est faible (BRADEN et al., I954), la rapidité de la dissolution de la zone pellucide par la pronase est décroissante de l'œuf non fécondé à l'œuf activé par choc électrique (émission partielle des GC) puis à l'œuf fécondé (MINTZ et GEARHART, I973).

Enfin, chez le Hamster, le blocage de la pénétrance de la zone pellucide aux spermatozoïdes par la substance des GC émise par des oufs fécondés ou activés par un choc électrique est supprimé par un inhibiteur de trypsine (GWATKIN et al., I973). Les GC possèderaient une activité trypsique qui agirait sur les récepteurs des spermatozoïdes dans la zone pellucide (HAR'TMANN et GWATKIN, I97I). Une situation analogue existerait dans le cas d'cufs d'oursins (VACQUier et al., I972).

La polyspermie spontanée ( $\mathrm{I}, 7 \mathrm{p}$. Ioo chez la Lapine) peut être due à un défaut d'émission des GC (leur persistance a été décrite dans plusieurs œufs polyspermiques par GulYAS, I974) ou à une rapidité insuffisante de la mise en fonction des systèmes de blocage (cas de présentation simultanée de deux spermatozoïdes), mais aussi à une absence de GC. Nous avons observé un œuf vierge jeune presque dépourvu de GC. Nous avons aussi rencontré des œufs vierges qui contiennent encore des amas de GC comme au stade de la synthèse de ces derniers (SzÖLLÖsI, I967).

\section{Distinction entre activation et blocage de la polyspermie}

Les faits rapportés, qui complètent ceux présentés par CHALMEI. (I962), montrent bien la dissociation entre un mécanisme local, la rupture des GC qui assure la monospermie, et le mécanisme général de l'activation qui conduit à la segmen- 
tation. Celui-ci peut être stimulé sans que nécessairement le premier le soit. On peut aussi obtenir un début de développement sans émission des GC chez des Echinodermes (Osanar, I960) et des Poissons (Iwamatsu, I965). Inversement, i1 a été montré que l'induction de la réaction corticale par le spermatozoïde (suivie de son élimination) n'induit pas nécessairement la segmentation (Nereis : L IL $_{1} I E$, IgI I ; GoODrICH, I920. Oursins : Hiramo'To, I962).

Signalons enfin que l'absence fréquente de gonflement du noyau spermatique dans les œufs activés puis fécondés, confirme la disparition du facteur cytoplasmique ovocytaire nécessaire à cette transformation, quand l'ovocyte vieillit ('THIBAULT, I973, tabl. 2).

Rę̧u poutr publication en octobre 1974.

P. S. : Après rédaction du présent article, nous avons pris connaissance d'une Communication qui confirme la rétention des GC dans les cufs de Lapines activés par un choc thermique (Gulyas, 1974, Am. J. Anat., 140, 577).

\section{REMERCIEMENTS}

Nous exprimons notre reconnaissance à. M. R. ScANDoLo pour le tirage des clichés et à Mlle M. E. Matet pour la dactylographie de ce mémoire.

\section{SUMMARY}

\section{CORTICAL REACTION AND INHIBI'TION OF POLYSPERMY IN THE RABBIT EGG}

Electron microscopy and biometric analysis are done on cortical granules (CG) of different types of rabbit eggs.

Results show that the $\mathrm{CG}$ remain present in the cortex of eggs ovulated during their fertile period ( 8 to Io hours after ovulation). This is also true of eggs artificially activated by cooling 24 hours at $10^{\circ} \mathrm{C}$, and reheating; these eggs remain fertilizable.

In fertilized eggs and those activated and then fertilized, the CG have almost disappeared. The penetration of fertilizing spermatozoa induces the loss of $C G$ and this cortical reaction inhibits fusion of supernumerary spermatozoa with the egg. The role of $C G$ in fertilization seems limited to this function.

The number of $C G$ tends to descrease in old oocytes, whether activated ot not. This explains the risk of polyspermy in late fertilization after ovulation.

\section{RÉFÉRENCES BIBLIOGRAPHIQUES}

Austin C. R., 1956. Activation of eggs by hypothermia in rat and hamsters. J. Exp. Biol., 33, 338-347. Austin C. R., I970. Ageing and reproduction : post-ovulatory deterioration of the egg. J. Reprod. Fert., suppl. 12, 39-54.

Austin C. R., Braden, A. W. H., 1954. Induction and inhibition of the second polar division in the rat egg and subsequent fertilization. A ustr. J. Biol. Sci., 7, 195-210. 
AUstin C. R., BRADen, A. W. H., 1956. Early reaction of the rodent egg to spermatozoon penetration. J. exp. Biol., 33, 358-365.

Barros C., Yanagimachi R., I97r. Induction of zona reaction in golden hamister eggs by cortical granule material. Nature, Lond., 233, 268.

Chalmel M. C., ig62. Possibilité de fécondation des ceufs de Lapine activés parthénogénétiquement. Ann. Biol. anim., Bioch. Biophys., 2, 279-297.

Chang M. C., r954. Development of parthenogenetic rabbit blastocysts induced by low temperature storage of unfertilized ova. J. exp. Zool., 125, I27-I 50.

Conkad K., Buckley J., Stambaugh R., I97I. Studies on the nature of the block to polyspermy in rabbit ova. J. Reprod. Fert., 27, 133-135.

Cooper G. W., BEDFord J. M., 197r. Charge density change in the vitelline surface following fertilization of the rabbit egg. J. Reprod. Fert., 25, 43I-436.

Fléchon J.-E., I97o. Nature glycoprotéique des GC de l'œuf de Lapine; mise en évidence par l'utilisation comparée de techniques cytochimiques ultrastructurales. J. Microscopie, 9, 22I-242.

Fraser L. R., Dandekar P. V., Gordon M. K., i972. Loss of cortical granules in rabbit eggs exposed to spermatozoa in vitro. J. Reprod. Fert., 29, 295-297.

Goodrich H. B., I920. Rapidity of activation in the fertilization of Nereis. Biol. Bull., 38, r96-20r.

Gulyas B. J., I974. Electron microscopic observations on advanced stages of spontaneous polyspermy in rabbit zygotes. Anat. Rec., 179, 285-296.

Gwatkin R. B. L., Williams D. T., Hartmann J. 1., Kniazuk M., 1973. The zona reaction of hamster and mouse eggs : production in vitro by a trypsin-like protease from cortical granules. $J$. Reprod. Fert., 32, 259-266.

Hartmann J. F., Gwatkin R. B. L., I97I. Alteration of sites on the mammalian sperm surface following capacitation. Nature, Lond., 234, 479-481.

Hiramoto Y., I962. An analysis of the mechanism of fertilization by means of microinjection of spermatozoa in sea urchin eggs. Exp. Cell. Res., 28, 323-334.

Iwamatsu T., I 965 . Effect of acetone on the cortical changes at fertilization of the egg of the Medaka orysias latipes. Embryologia, 9, I-I2.

Lillie F. R., I9II. Studies of fertilization in Nereis I. The cortical changes in the egg. II. Partial fertilization. J. Morphol., 22, 36 I-393.

LoNGo J. F., I974 a. Ultrastructural changes in rabbit eggs aged in vivo. Biol. Reprod., 11, 22-39.

LONGo J. F., r944 $b$. An ultrastructural analysis of spontaneous activation of hamster eggs aged in vivo. Anat. Rec., 179, 27-56.

Merchant H., Chang M. C., I97I. An electron microscopic study of mouse eggs matured in vivo and in vitro. Anat. Rec., 171, $2 \mathrm{I}-38$.

Mintz B., Gearhart J. D., I973. Subnormal zona pellucida change in parthenogenetic mouse embryos. Develop. Biol., 31, I $78-184$.

OsanaI K., 1960. Development of the sea urchin egg with the inhibited breakdown of the cortical granules. Sci. Rep. Tohoku Uniu. Ser. IV, 26, 77-87.

Peluso J. J., Butcher R. L., 1974. The effect of follicular aging on the ultrastructure of the rat oocyte. Fert. Steril., 25, 494-502.

PIKo L., r969. Gamete structure and sperm entry in Mammals. In : Fertilization, 2, 235-403, METZ C. B. and Monroy A., Acad. Press N.Y.

Szölzös D D., I967. Development of cortical granules and the cortical reaction in rat and hamster eggs. Anat. Rec., 159, 431-446.

Szöllösr D., I97r. Morphological changes in mouse eggs due to aging in the fallopian tube. Am. J. Anat., 130, 209-226.

TARKovski A. K., Wrowowska A., Nowicka J., I970. Experimental parthenogenesis in the mouse. Nature, Lond., 226, Iن்2-I65.

Тнiвaulx C., 1972. Physiology and physiopathology of the fallopian tube. Int. J. Fertil., 17, I-I3.

Thibault C., I973. Experimental approaches to normal and abnormal mammalian oocyte maturation and ageing, in: Les accidents chromosomiques de la reproduction, I. N. S. E. R. M., Bové et THIBAULT ed., $233-240$.

VAcQuier V. D., Tegner M. J. EPEL D., 1972. Protease activity establishes the block against polyspermy in sea eggs. Nature, Lond., 240, 352-353.

Yanagimachi R., Chang M. C., I96I. Fertilizable life of golden hamster ova and their morphological changes at the time of losing fertilizability. J. exp. Zool., 148, I85-203. 
PLANCHES 


\section{PLANCHE I}

\section{FIG. I}

Oeuf "activé in vivo ", $6 \mathrm{~h}$ après réchauffement. De nombreux $\mathrm{GC}$ sont distribués de manière relativement homogène dans le cortex; quelques-uns sont situés à l'intérieur du cytoplasme $\times 7000$ ).

FIG. 2

Oeuf "activé in vitro ", $30 \mathrm{mn}$ après réchauffement. Répartition similaire des GC. L,e contenu des vésicules de reticulum endoplasmique est modifié par le séjour in vitro mais reprend un aspect habituel in vivo (fig. I) ( $\times 7$ ooo). 

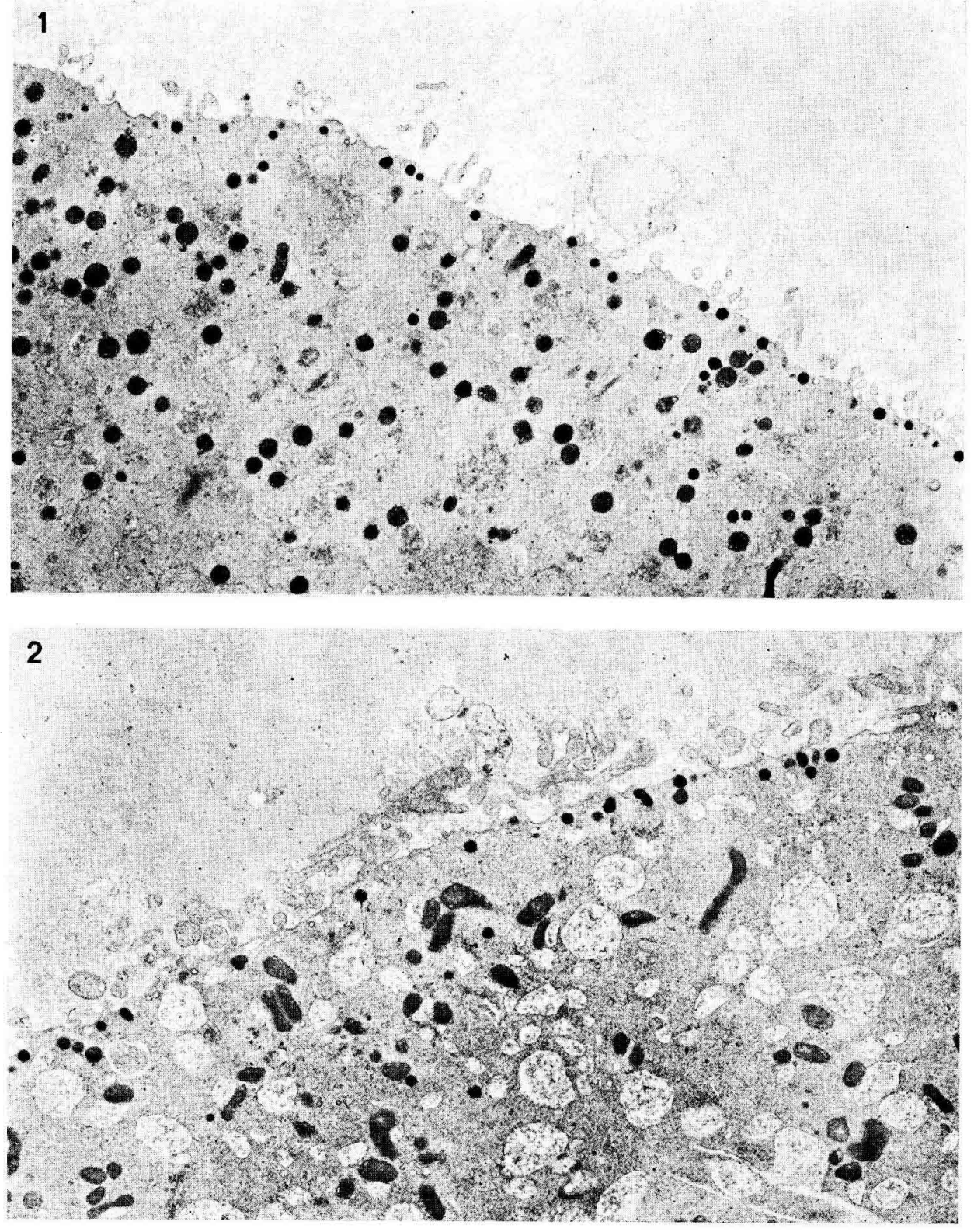

J. E. FLECHON, D. HUNEAU, A. SOLARI et C. THIBAULT 


\title{
PLANGHE II
}

\author{
Fig. 3
}

Euf "activè in vitro, $1 \mathrm{~h}$ après réchauffement. Un exemple d'hétérogénéité locale de la distribution des GC dans le cortex. $(\times 11.500)$.

FIG. 4

Guf a activè in vivon, $6 \mathrm{~h}$ après rềchauffement. Distribution très régulière des $\mathrm{GC}$ dans le cortex. $(\times 11.500)$. 

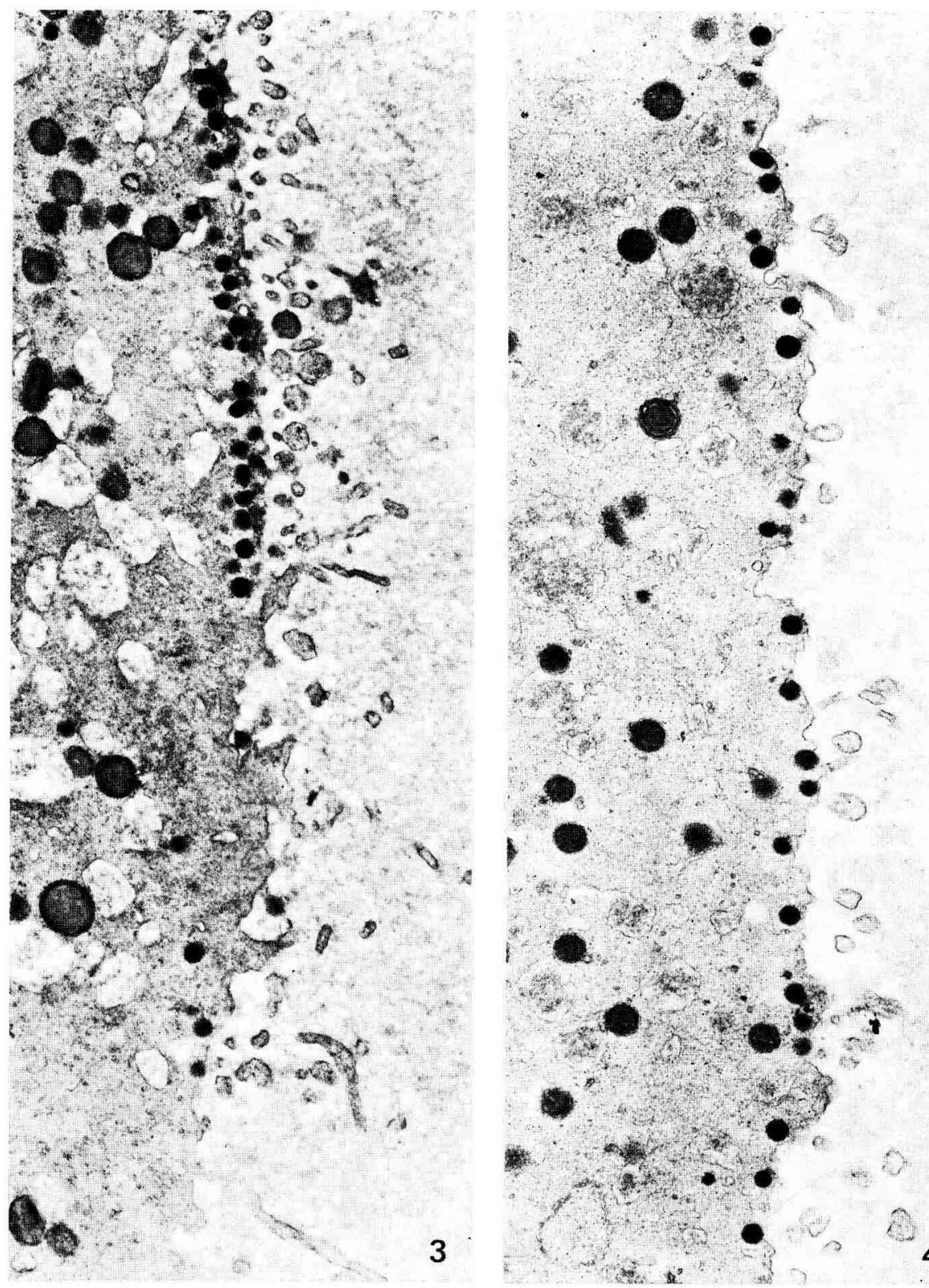


\title{
PLANCHE III
}

\author{
FIG. 5
}

Euf "activé et fécondé in vivo ", $4 \mathrm{~h} 30$ après transplantation. La pièce principale du spermatozoïde fécondant est localisée sous la membrane plasmique. Le cortex ne contient pas de GC. $(\times 5.200)$.

FIG. 6

Euf "activé et fécondé in vivo ", $4 \mathrm{~h} 30$ après transplantation. L'émission des GC s'est produite. Le cytoplasme périphérique de l'œuf contient une pièce intermédiaire. $(\times 17.500)$. 


\section{5}

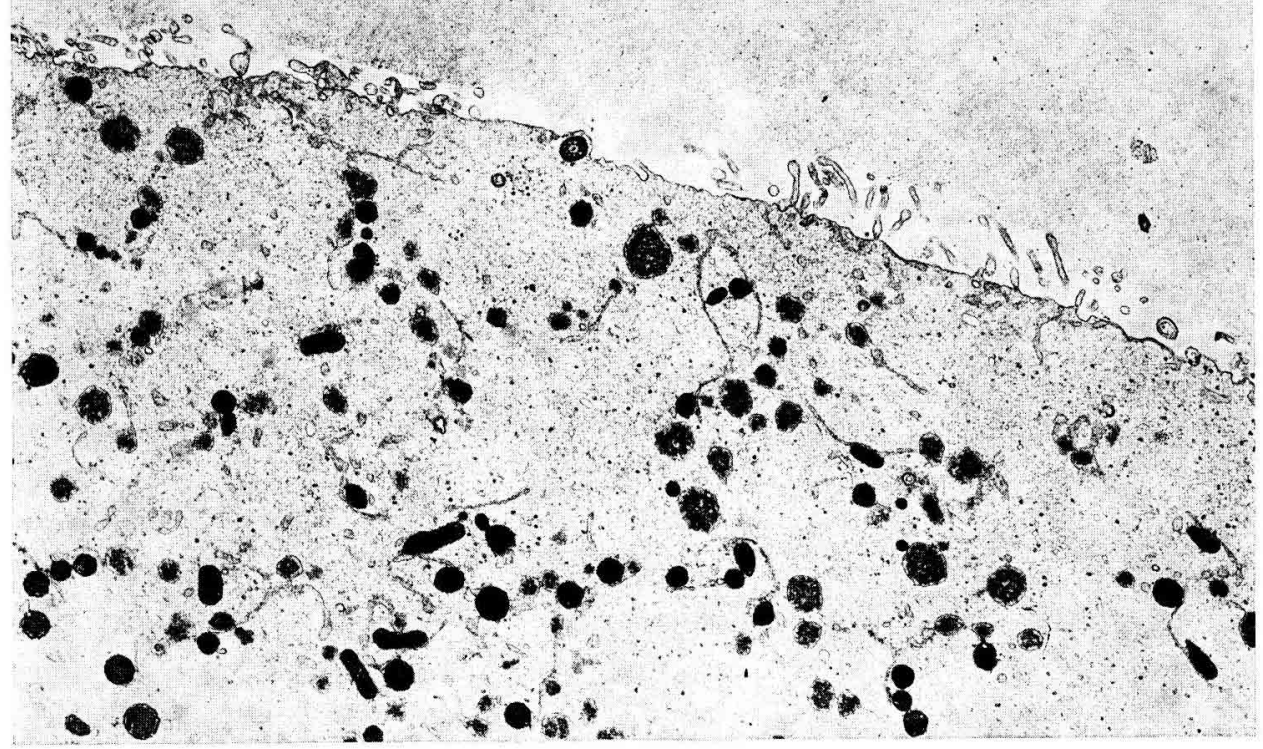

6

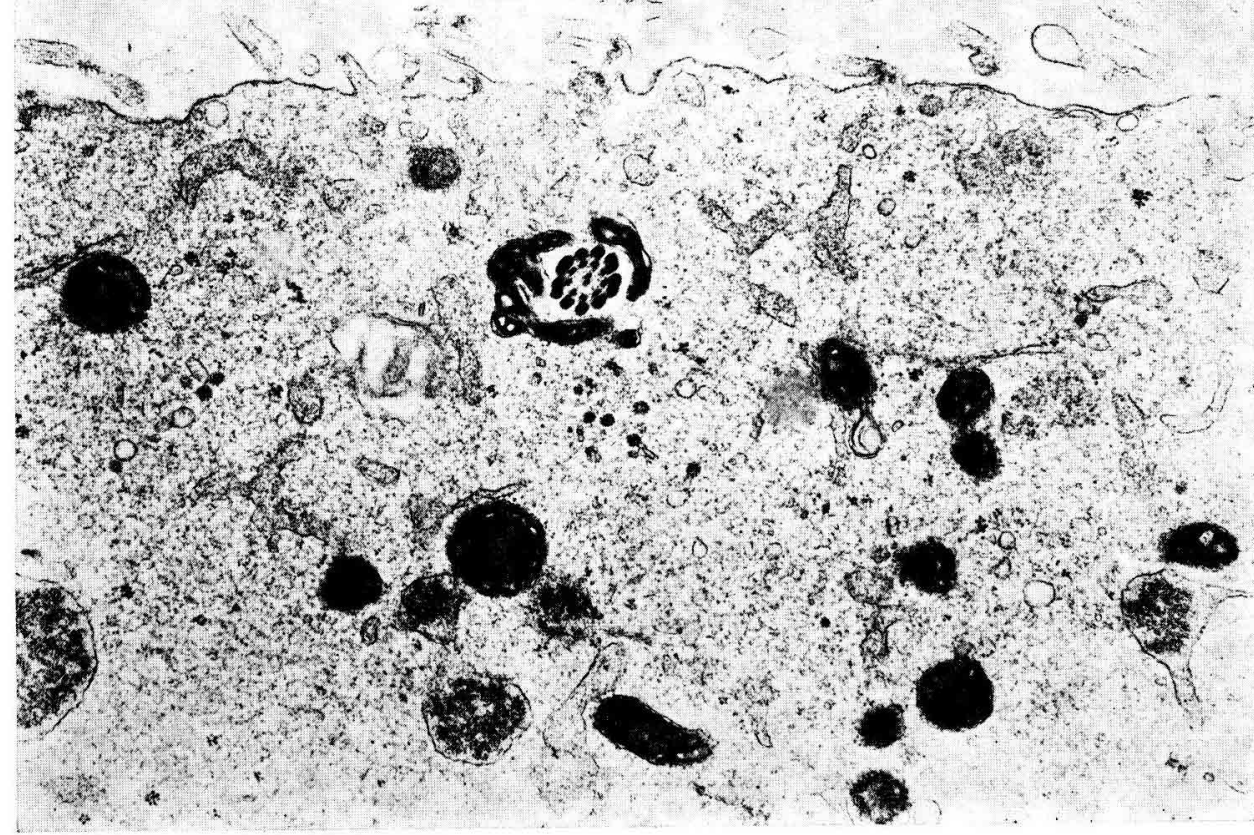

J. E. FLECHON, D. HUNEAU, A. SOLARI et C. THIBAULT 